\title{
High-Power Single-Mode Submonolayer Quantum-Dot Photonic Crystal Vertical-Cavity Surface-Emitting Lasers
}

\author{
Fang-I. Lai, H. P. D. Yang, G. Lin, I.-Chen Hsu, Jui-Nung Liu, N. A. Maleev, S. A. Blokhin, \\ H. C. Kuo, Senior Member, IEEE, and Jim Y. Chi, Senior Member, IEEE
}

\begin{abstract}
A high-power, wide operation-range singlefundamental-mode InGaAs submonolayer (SML) quantum dot (QD) photonic crystal vertical-cavity surface-emitting laser (VCSEL) for fiber-optic applications was demonstrated. Singlefundamental-mode with a threshold current $\left(I_{t h}\right)$ of $0.9 \mathrm{~mA}$ and maximum continue-wave $(\mathrm{CW})$ output power of $3.8 \mathrm{~mW}$ at $28 \mathrm{~mA}$ has been achieved in the $990 \mathrm{~nm}$ range. A side-mode suppression ratio (SMSR) larger than $35 \mathrm{~dB}$ has been observed over the entire current operating range. The far field measurement shows that the beam divergence angle remains very small and almost unchanged between $6.7-6.9^{\circ}$, with the operation range increasing from 3 to $20 \mathrm{~mA}$.
\end{abstract}

Index Terms-Divergence angle, quantum-dot, single-mode, submonolayer (SML), vertical-cavity surface-emitting laser (VCSEL).

\section{INTRODUCTION}

V ERTICAL-CAVITY surface-emitting lasers (VCSELs) are becoming attractive low-cost, high-performance light sources for various applications including local area networks (LANs), fiber-optic communication systems, data storage, optical computing and different sensors [1] for their unique characteristics such as circular laser beam, low threshold current, low power consumption, high modulation bandwidth, easy polarization control, capability of fabricating dense 2-D laser arrays, and the testing of wafer levels. For short-distance optical interconnects in the chip-to-chip, backplane, and board-to-board fiber transmission, VCSELs emission in the $980 \mathrm{~nm}$ range are reliable and can be compatible with the $1300 \mathrm{~nm}$ receiver [2]. For high-speed optical data communication applications, single

Manuscript received October 31, 2006; revised August 7, 2007. This work was supported in part by the Nanophotonics Project, MOEA, Taiwan, and SANDIA (NMP4-CT-2004-500101), and in part by the National Science Council of Republic of China (ROC), Taiwan, under Contract 95-2622-E-231-006-CC3.

F.-I. Lai is with the Department of Electrical Engineering, Yuan Ze University, Jung-Li, Taoyuan 320, Taiwan, R.O.C. (e-mail: filai@saturn.yzu.edu.tw).

H. P. D. Yang and J. Y. Chi are with theNanophotonic Center, Industrial Technology Research Institute, Hsinchu 310, Taiwan, R.O.C. (e-mail: hpyang@itri.org.tw; chij@itri.org.tw).

G. Lin is with the Department of Electronics Engineering, National Chiao Tung University, Hsinchu 300, Taiwan, R.O.C. (e-mail: graylin@mail.nctu.edu.tw).

I.-C. Hsu, J.-N. Liu, and H. C. Kuo are with the Institute of ElectroOptical Engineering, National Chiao Tung University, Hsinchu 300 Taiwan, R.O.C. (e-mail: baddin58@yahoo.com.tw; 1.y.n@yahoo.com.tw; hckuo@faculty.nctu.edu.tw).

N. A. Maleev and S. A. Blokhin are with the A. F. Ioffe Physico-Technica Institute, Russian Academy of Sciences, St. Petersburg 194021, Russia (e-mail: nmaleev@yandex.ru; blokh@mail.ioffe.ru).

Digital Object Identifier 10.1109/JSTQE.2007.906352 fundamental mode operation of VCSEL sources is beneficial because they can be coupled into the central low-modal dispersion region of a multimode fiber [3] or a single-mode fiber with a smaller core diameter. Traditional oxide confined VCSEL using small oxide aperture below about $4 \mu \mathrm{m}$ diameter operate in the fundamental transverse mode. However, the small aperture leads to low output power and large resistance, which limits the modulation bandwidth and degrades the high-speed performance of the device. Moreover, the lifetime of the oxide VCSEL also decreases proportionally to the diameter of the oxide aperture, even when the device is operated at a reduced current [4]. Other methods to achieve single fundamental mode operation include the increase of higher order mode loss by surface-relief etching [5], hybrid-oxide-implanted VCSELs [6], [7], and twodimensional triangular holey structure [8], [9]. Recently, a 2-D photonic crystal structure fabricated on the VCSEL surface has been used as a control method of transverse modes. Singlemode output was realized from larger aperture photonic crystal VCSELs [10]-[12].

The stacked submonolayer growth of quantum dots (SML QDs) presents an alternative approach to Stranski-Krastanow (SK) method, which contain InGaAs QD sheets separated by AlGaAs spacers [13] of growing self-assembled QDs for the $0.85-1.3 \mu \mathrm{m}$ wavelength range for lasers and amplifiers. The SML QDs can grow with higher density and high uniformity, which can provide high material and modal gain and result in high-power lasing performance, and surpass the SK QD laser [13], [14]. Recently, single-mode SML QD VCSELs have been demonstrated with $1 \mu \mathrm{m}$ in diameter oxide confined aperture to realize high modulation bandwidth [15]. However, due to the small oxide aperture, the output power is around $1 \mathrm{~mW}$. In this paper, we report our results on using 2-D photonic crystal structure on the InGaAs SML QD VCSELs to achieve larger aperture single-transverse-mode operation with high output power and high side-mode suppression ratio (SMSR).

\section{EXPERIMENT}

The epitaxial layers of the InGaAs SML QD photonic crystal VCSEL wafers were grown on 3 in $^{+}{ }^{+}$GaAs $\left(\begin{array}{lll}0 & 0 & 1\end{array}\right)$ substrates by molecular beam epitaxy (MBE) in Riber 49 chamber. The bottom distributed Bragg reflector (DBR) consists of a 33-pair n-type (Si-doped) quarter-wave stack $(\lambda / 4)$ of $\mathrm{Al}_{0.9} \mathrm{Ga}_{0.1}$ As/GaAs. The top DBR consists of a 20-period p-type (carbon-doped) $\mathrm{Al}_{0.9} \mathrm{Ga}_{0.1} \mathrm{As} / \mathrm{GaAs}$ quarter-wave stack. 


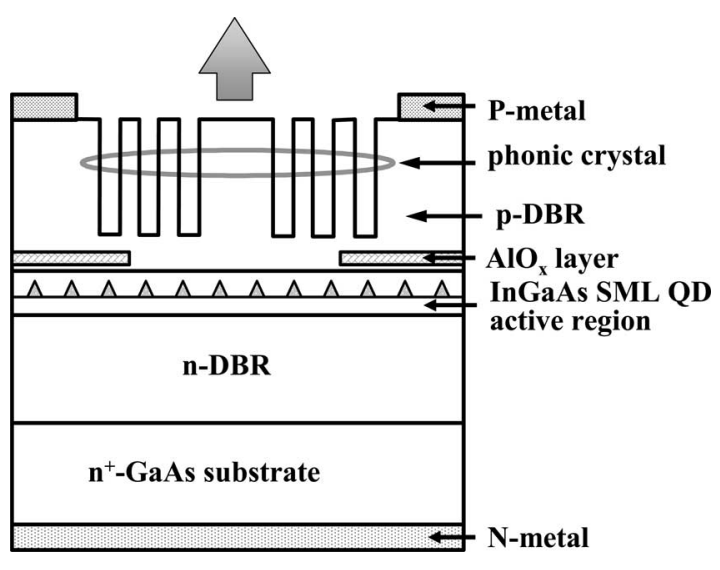

Fig. 1. Schematic of InGaAs SML QD photonic crystal VCSEL. The hole etching depth of the photonic crystal structure is 16 pairs out of the 20 pairs top DBR being etched off.

Above that is a heavily doped p-type GaAs contact layer. The undoped $1 \lambda$-cavity contains three InGaAs SML QDs layers, separated by GaAs barrier layers. Each of the InGaAs SML QDs layers is formed by alternate deposition of InAs ( $<1 \mathrm{ML})$ and GaAs. The current confinement of the device was done by the selectively oxidized $\mathrm{AlO}_{x}$ tapered aperture. Firstly, mesas with diameters varying from 50 to $68 \mu \mathrm{m}$ were defined by reactive ion etch (RIE). The inner diameter of p-contact ring was $10 \mu \mathrm{m}$ larger than the oxide aperture and formed on the top of the p-contact layer. The AlAs layer within the $\mathrm{Al}_{0.9} \mathrm{Ga}_{0.1}$ As confinement layers was selectively oxidized to $\mathrm{AlO}_{x}$. The oxidation depth was about 16-17 $\mu \mathrm{m}$ toward the center from the mesa edge so that the resulting oxide aperture varied from 16 to $36 \mu \mathrm{m}$ in diameter. The oxide aperture was introduced at a minimum of the optical field in order to reduce the lateral optical loss. The $\mathrm{AlO}_{x}$ layer was also used to reduce the leakage current. The n-contact was formed at the bottom of the $\mathrm{n}^{+}$-GaAs substrate. After that, triangular lattice patterns of photonic crystal with a single-point defect in the center were defined within the p-contact ring using photolithography, and were etched through the p-type DBR using RIE. The lateral index around a single defect can be controlled by the hole diameter $(\alpha)$-to-lattice constant $(\Lambda)$ ratio and etching depth [11]. This ratio $(\alpha / \Lambda)$ is 0.5 ; the lattice constant $\Lambda$ is $5 \mu \mathrm{m}$ in the photonic crystal VCSEL, and the etching depth of the holes is about 16-pair thick into the 20-pair top DBR layer. The photonic crystal parameters are chosen not only to provide reasonable lateral index guiding and loss mechanism for the lasing output, but also to leave suitable conduction paths ( $\sim 2.5 \mu \mathrm{m}$ width) for the injection current to flow through the photonic crystal holes, and then, toward the central laser emission region. The device structure is shown in Fig. 1. By using two types of apertures in this device, we decouple the effects of the current confinement from the optical confinement. The $\mathrm{AlO}_{x}$ layer is used to confine the current flow, while the single-point defect (approximately $\geq 10 \mu \mathrm{m}$ in diameter) photonic crystal is used to confine the optical mode. In order to clarify the effect of the photonic crystal index-guiding layer, an oxide-confined VCSEL (oxide aperture $=18 \mu \mathrm{m}$ in diameter without photonic crystal structure) was also fabricated for comparison.

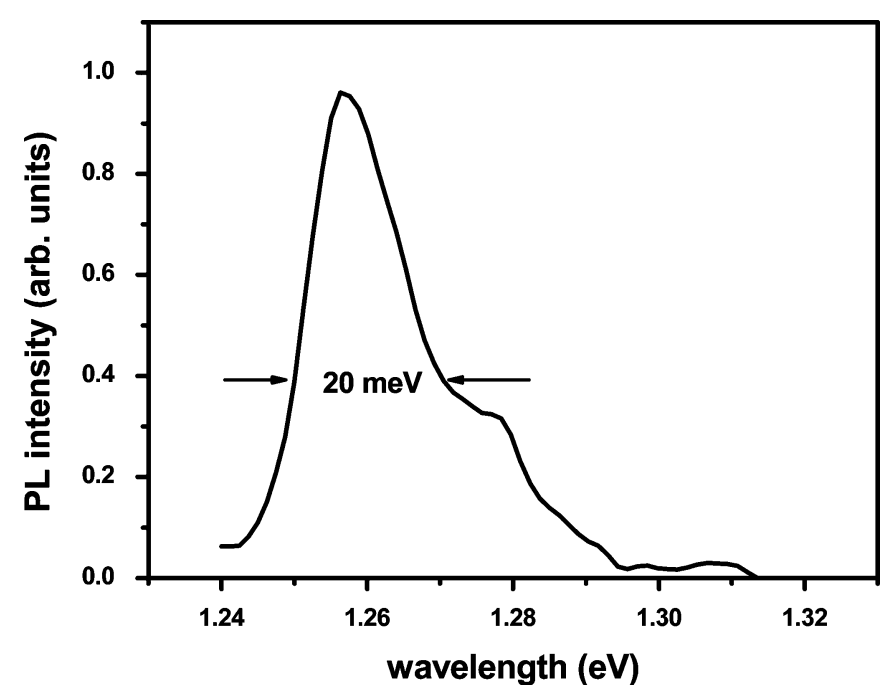

Fig. 2. Room temperature photoluminance spectrum of top DBR removed SML QD VCSEL wafer.

\section{RESULTS AND DISCUSSIONS}

The small part of the SML QD VCSEL wafer was removed from the top DBR by RIE and performed the photoluminance (PL) measurement to investigate the material characteristics of the SML QDs. The PL measurement was carried out by the $532 \mathrm{~nm}$ line of an $\mathrm{Nd}: \mathrm{YVO}_{4}$ laser with a power density of $265 \mathrm{~mW} / \mathrm{cm}^{2}$, and the emission from the samples was dispersed by a monochromator, and was detected by using a thermoelectrically cooled InGaAs detector. The PL spectrum of SML QD at room temperature was depicted in Fig. 2, and the peak of emission spectrum is $1.257 \mathrm{eV}$ in ground state, which corresponds to $986.5 \mathrm{~nm}$. The spectrum shows very narrow full-width at half-maximum (FWHM) of about $20 \mathrm{meV}$, representing the highly uniformity of QDs size. The InGaAs SML QD VCSEL devices without photonic crystal were performed $\mathrm{CW}$ lightcurrent-voltage $(L-I-V)$ output measurement to characterize the performance of the device, and shown in Fig. 3(a). The VCSEL with a mesa of $50 \mu \mathrm{m}$ in diameter and oxide aperture of $18 \mu \mathrm{m}$ in diameter shows a threshold current $\left(I_{\mathrm{th}}\right)$ of $1 \mathrm{~mA}$ and a peak power of $12.2 \mathrm{~mW}$ at $30 \mathrm{~mA}$. The differential series resistance is approximately $100 \Omega$ at $12 \mathrm{~mA}$. Fig. 3(b) shows the $\mathrm{CW}$ $L-I-V$ output of the photonic crystal VCSEL. The near-field image of the photonic crystal VCSEL operating at $4 \mathrm{~mA}$ is also shown in the inset. The mesa of the photonic crystal VCSEL is also $50 \mu \mathrm{m}$ in diameter, and the oxide aperture is of the same size as the device in Fig. 3(a). The photonic crystal VCSEL emits a maximum power of $3.8 \mathrm{~mW}$ at $28 \mathrm{~mA}$ and exhibits single-mode operation throughout the whole current range of operation. Near-field imaging of the output mode remains to be fundamental $\mathrm{TEM}_{00}$ mode at the center of the photonic crystal structure throughout the current operating range. The $I_{\mathrm{th}}$ of the photonic crystal VCSEL is $0.9 \mathrm{~mA}$. The differential series resistance of the photonic crystal VCSEL is approximately $125 \Omega$ at $12 \mathrm{~mA}$. The $I-V$ characteristics exhibit slightly higher series resistance for the photonic crystal VCSEL, which would be mainly due to blocking of the current flow in the region by 

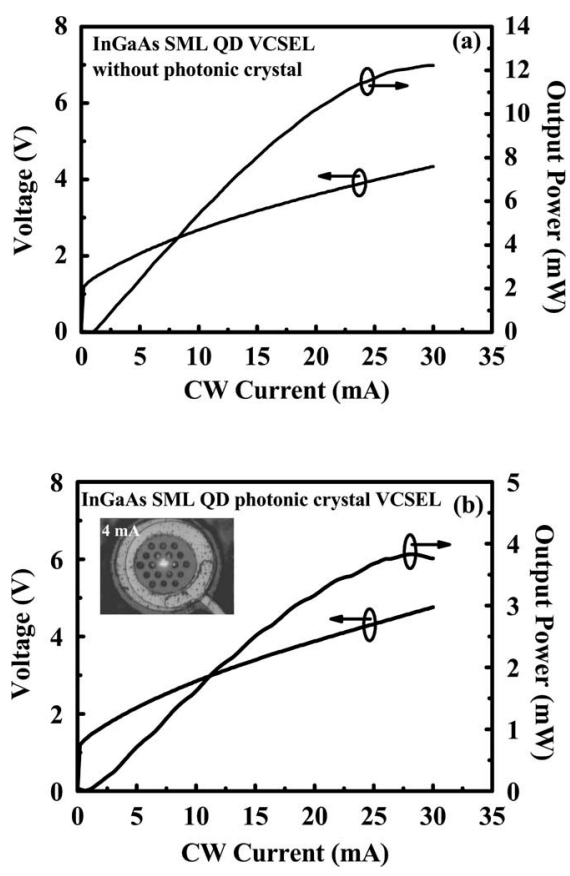

Fig. 3. CW $L-I-V$ characteristics and near-field image (inset) of InGaAs SML QD VCSEL. (a) Without photonic crystal. (b) With photonic crystal. The ratio $\alpha / \Lambda$ is 0.5 and the lattice constant $\Lambda$ is $5 \mu \mathrm{m}$. The oxide aperture is $18 \mu \mathrm{m}$ in diameter.
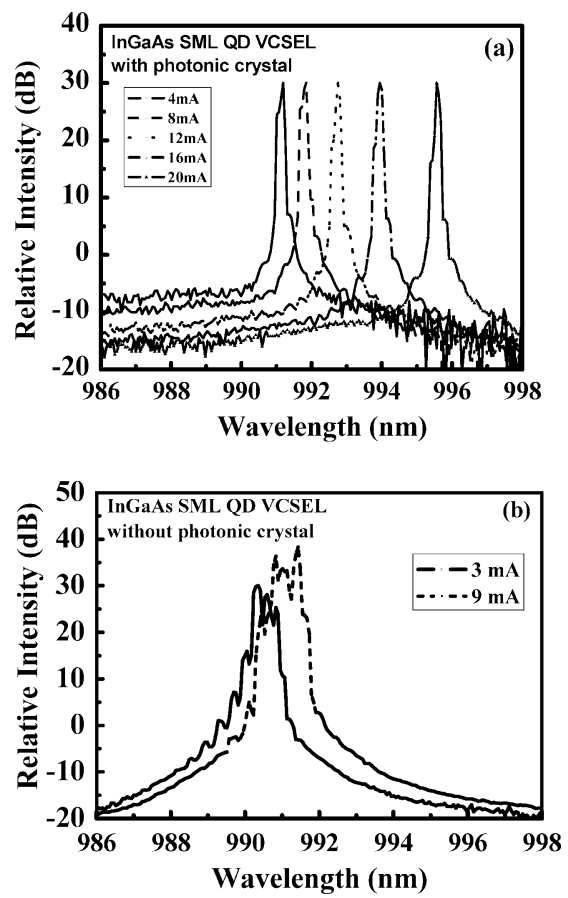

Fig. 4. Spectra of the InGaAs SML QD VCSEL. (a) With photonic crystal structure. (b) Without photonic crystal structure. The ratio $\alpha / \Lambda$ is 0.5 and the lattice constant $\Lambda$ is $5 \mu \mathrm{m}$. The oxide aperture is $18 \mu \mathrm{m}$ in diameter.

photonic crystal holes. Lasing spectra of the photonic crystal VCSEL are shown in Fig. 4(a), confirming single-mode operation within the wide operation current from 1 to $28 \mathrm{~mA}$. The peak lasing wavelengths are red shifted from 991 to $995.5 \mathrm{~nm}$ when

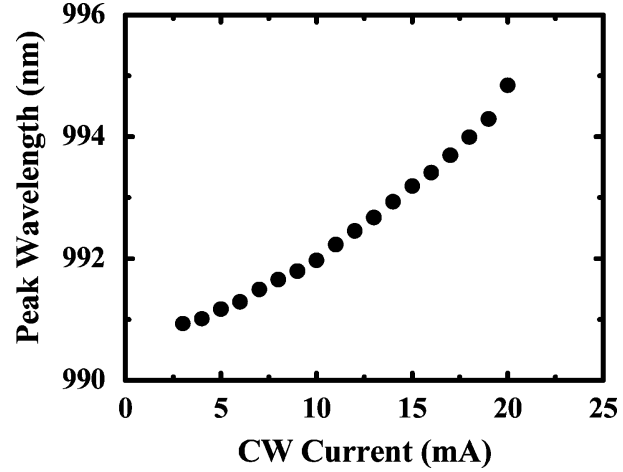

Fig. 5. Current-dependent lasing peak wavelength of the InGaAs SML QD photonic crystal VCSEL.

bias current ranges from 4 to $20 \mathrm{~mA}$. The difference between peak of PL and lasing spectrum results from the detuning of gain spectrum and Fabry-Perot dip of DBR. The photonic crystal VCSEL exhibits an SMSR $>35 \mathrm{~dB}$ throughout the current range. The peak wavelength versus injection current plot of the InGaAs SML QD photonic crystal VCSEL is shown in Fig. 5. The peak lasing wavelength increases monotonically with the increasing injection current. The increase in wavelength with increasing injection current is mainly due to the increase in junction temperature. The rate of peak wavelength increase with biasing current $(\Delta \lambda p / \Delta I)$ is approximately $0.15 \mathrm{~nm} / \mathrm{mA}$ from 3 to $10 \mathrm{~mA}$, and the rate approximately is $0.26 \mathrm{~nm} / \mathrm{mA}$ from 10 to $20 \mathrm{~mA}$. The rate of peak wavelength is different for different current range, which is mainly because of thermal conduction of the device and the measurement system. The junction temperature of the device does not increase linearly with current. The lasing in QDs is believed to be ground-state transition since all the measured spectra are single mode for the photonic crystal VCSELs. No side mode corresponding to an excited state transition was observed at higher currents. For comparison, lasing spectra of the InGaAs QD VCSEL without photonic crystal holes is shown in Fig. 4(b). It is clear that multiple-mode operation as the driving current is increased above $I_{\text {th }}$. The InGaAs SML QD VCSEL without photonic crystal shows multiple transverse mode characteristics with a broader wavelength span. The nearfield images of the VCSEL without photonic crystal are shown in Fig. 6. The near-field image of the VCSEL at $3 \mathrm{~mA}$ [Fig. 6(a)] was taken with light illumination to show the relative position of the lasing area with respect to the p-contact ring. The lasing area is annular in shape ( $15 \mu \mathrm{m}$ in diameter) with lower intensity in the central region. The near-field images at 9 and $20 \mathrm{~mA}$ [Fig. 6(b) and (c)] show lasing areas with higher intensity at the center. The lasing area of the device at $20 \mathrm{~mA}$ is about $18 \mu \mathrm{m}$ in diameter, which is close to the diameter of the oxide aperture. All the near-field images show that the laser beam is of multimode characteristics because of the larger oxide aperture (approximately $18 \mu \mathrm{m}$ in diameter) of the device. With the same oxide aperture size, the VCSELs with a photonic crystal structure clearly show much better optical confinement. Fig. 7 shows the near-field image of the photonic crystal VCSEL at $10 \mathrm{~mA}$. The laser output is a fundamental $\mathrm{TEM}_{00}$ mode at the center of the photonic crystal structure. The $\mathrm{TEM}_{00}$ mode is rounded 

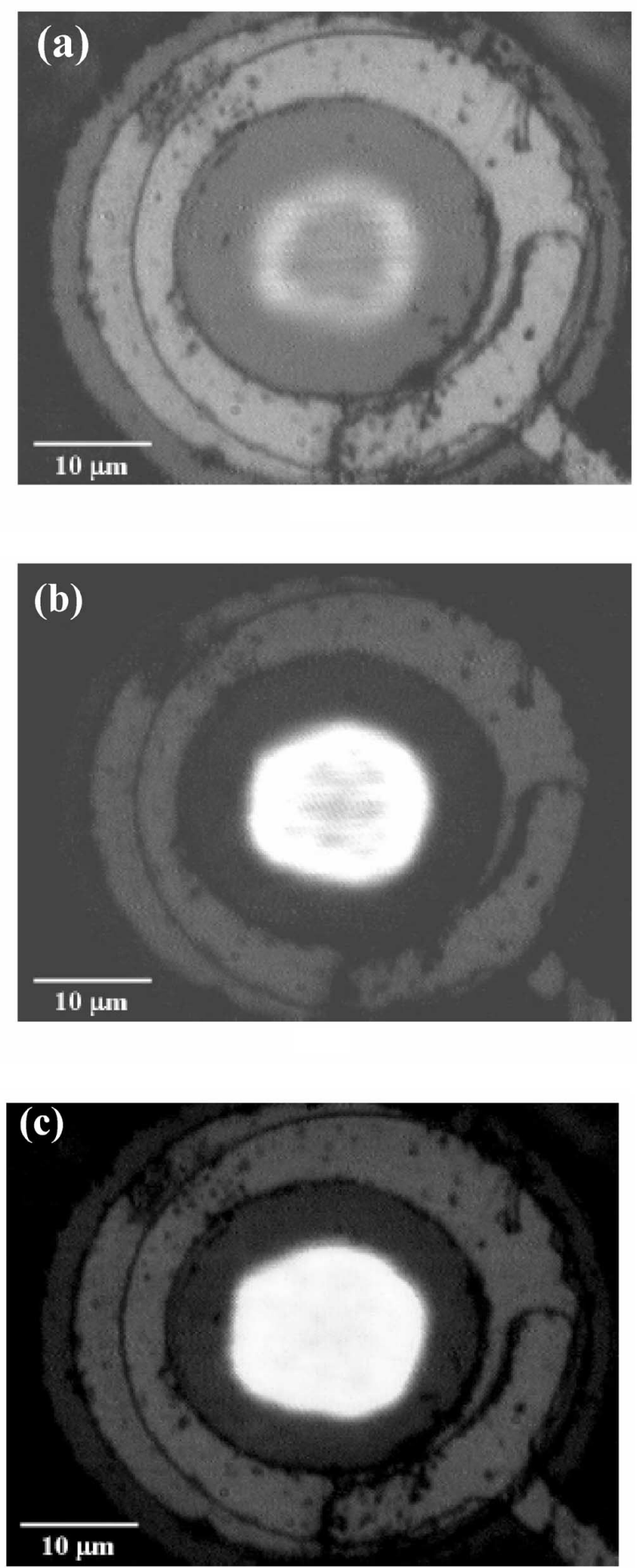

Fig. 6. Near-field images of the InGaAs SML QD VCSEL without photonic crystal. (a) At $3 \mathrm{~mA}$. (b) At $9 \mathrm{~mA}$. (c) At $20 \mathrm{~mA}$. The oxide aperture is $18 \mu \mathrm{m}$ in diameter.

in shape with a diameter of approximately $4.3 \mu \mathrm{m}$. Smaller lasing spots emitting from the nearby photonic crystal holes with lower intensity were also observed. These lasing spots are mainly the scattered and diffracted laser beams from the $\mathrm{TEM}_{00}$

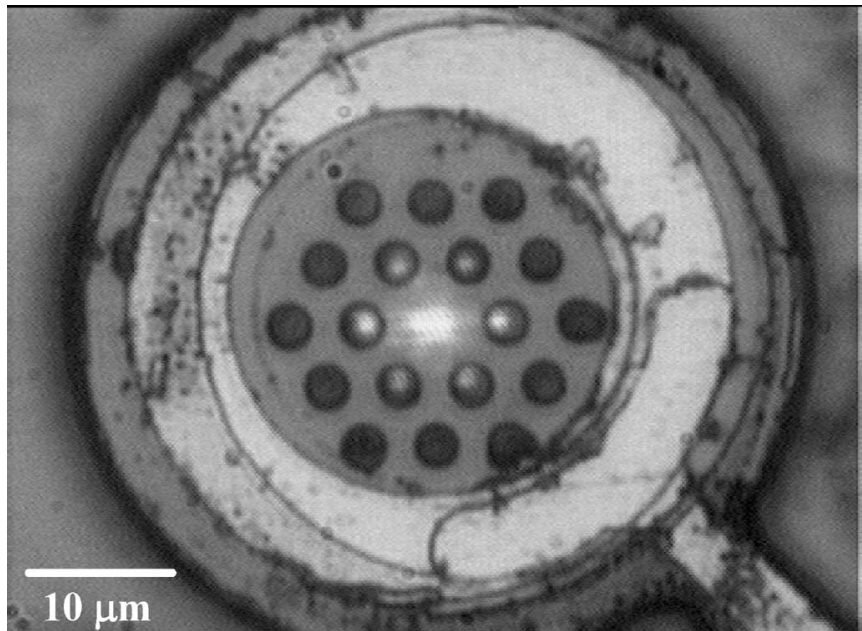

Fig. 7 Near-field image of the InGaAs SML QD photonic crystal VCSEL at $10 \mathrm{~mA}$. The oxide aperture is $18 \mu \mathrm{m}$ in diameter.

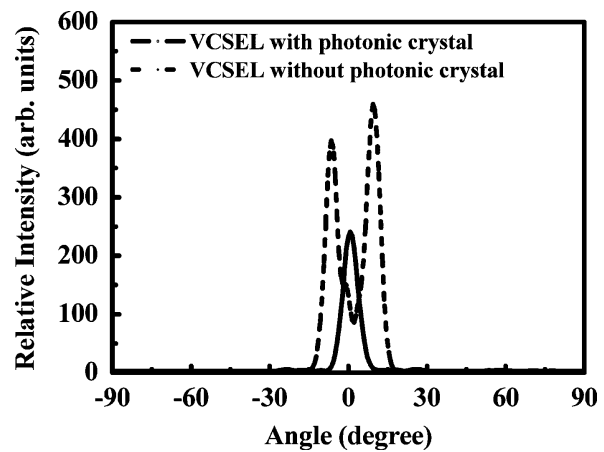

Fig. 8. Beam profiles of InGaAs SML QD VCSEL with and without photonic crystal measured at $10 \mathrm{~mA}$. The oxide aperture is $18 \mu \mathrm{m}$ in diameter.

beam at the center, which are all emitting with the same lasing wavelength.

The beam profile results obtained from far-field beam pattern measurements at a bias current of $10 \mathrm{~mA}$ are shown in Fig. 8 . The beam divergence angle is the FWHM of the beam profile. The beam divergence angle of VCSEL without photonic crystal is $21.5^{\circ}$. The two laser emission lobes correspond to multimode laser emission that is annular in shape, as observed by the near-field imaging. This annular shaped laser emission has a low intensity at the center. The lasing output consists of many higher-order modes because of the large oxide aperture (18 $\mu \mathrm{m}$ in diameter). For photonic crystal VCSEL, the beam divergence angle is very small, about $6.7^{\circ}$. Fig. 9 shows the current-dependent divergence angles of the InGaAs SML QD photonic crystal VCSEL and InGaAs SML QD VCSEL without photonic crystal. For the InGaAs SML QD VCSEL without photonic crystal, the divergence angle increases monotonically from $17.2^{\circ}$ to $22.8^{\circ}$, with current increasing from 3 to $13 \mathrm{~mA}$. The detector, which measured divergence angle, was saturated when bias current was over $13 \mathrm{~mA}$. For the InGaAs SML QD photonic crystal VCSEL, the beam divergence angle remains very small and almost unchanged between $6.7^{\circ}$ and $6.9^{\circ}$, with respect to increasing current from 3 to $20 \mathrm{~mA}$. This divergence 


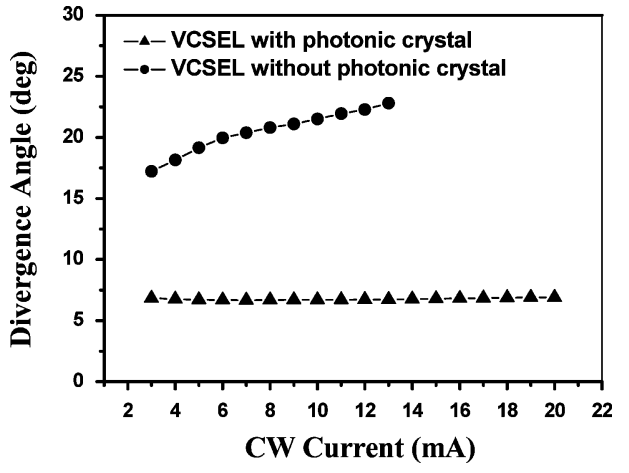

Fig. 9. Current-dependent beam divergence angle of InGaAs SML QD photonic crystal VCSEL $(\boldsymbol{\Delta})$ and InGaAs SML QD VCSEL without photonic crystal $(\bullet)$. The oxide aperture is $18 \mu \mathrm{m}$ in diameter.

angle obviously indicates that the laser beam is well confined by the photonic crystal structure of the device. The photonic crystal structure not only laterally confines the lasing mode, but also provides a waveguide for the $\mathrm{TEM}_{00}$-lasing-mode output with very small divergence.

\section{CONCLUSION}

We have demonstrated a high-power, single-mode InGaAs SML QD photonic crystal VCSEL with SMSR $>35 \mathrm{~dB}$ throughout the current operating range. The maximum output power is $3.8 \mathrm{~mW}$, which is the highest reported for the photonic crystal VCSELs. The beam divergence angle remains very small and almost invariant around $6.8^{\circ}$, within the operation current range from 3 to $20 \mathrm{~mA}$. The beam profile and near-field image of the photonic crystal VCSEL indicates that the laser beam is well confined by the photonic crystal structure of the device. The present results indicate that a VCSEL using an oxide layer for current confinement and photonic crystal for optical confinement is a promising approach to achieve single-mode operation of VCSEL.

\section{ACKNOWLEDGMENT}

The authors would like to thank Dr. A. R. Kovsh of NL Nanosemiconductor GmbH in Germany for providing the InGaAs SML QD-VCSEL epitaxial wafers, and Prof. S. L. Chuang of the University of Illinois, UrbanaChampaign, Prof. S. C. Wang and Prof. S. Chi of the National Chiao Tung University, Hsinchu, Taiwan, and Prof. Koyama of the Tokyo Institute of Technology, Tokyo, for their helpful discussion.

\section{REFERENCES}

[1] C. W. Wilmsen, H. Temkin, and L. A. Coldren, Vertical-Cavity SurfaceEmitting Lasers: Design, Fabrication, Characterization, and Applications. Cambridge, U.K.: Cambridge Univ. Press, 1999.

[2] K. J. Ebeling, R. Michalzik, R. King, P. Schnitzer, D. Wiedenmann, R. Jäger, C. Jung, M. Grabherr, and M. Miller, "Application of VCSELs for optical interconnects," in Proc. 24th Eur. Conf. Opt. Commun., Madrid, Spain, Sep. 20-24, 1998. New York; IEEE, vol. 3, pp. 27-31.
[3] P. Pepeljugoski, D. Kuchta, Y. Kwark, P. Pleunis, and G. Kuyt, "15.6$\mathrm{Gb} / \mathrm{s}$ transmission over $1 \mathrm{~km}$ of next generation multimode fiber," IEEE Photon. Technol. Lett., vol. 14, no. 5, pp. 717-719, May 2002.

[4] B. M. Hawkins, R. A. III Hawthorne III., J. K. Guenter, J. A. Tatum, and J. R. Biard, "Reliability of various size oxide aperture VCSELs," in Proc. 52nd Electron. Comp. \& Technol. Conf., 2002, pp. 540-550.

[5] A. Haglund, J. S. Gustavsson, J. Vukusic, P. Modh, and A. Larsson, "Single fundamental-mode output power exceeding $6 \mathrm{~mW}$ from VCSELs with a shallow surface relief," IEEE Photon. Technol. Lett., vol. 16, no. 2, pp. 368-370, Feb. 2004.

[6] E. W. Young, K. D. Choquette, S. L. Chuang, K. M. Geib, A. J. Fischer, and A. A. Allerman, "Single-transverse-mode vertical-cavity lasers under continuous and pulsed operation," IEEE Photon. Technol. Lett., vol. 13, no. 9, pp. 927-929, Sep. 2001.

[7] F.-I. Lai, T. H. Hsueh, Y. H Chang, H. C. Kuo, S. C. Wang, L. H. Laih, C. P. Song, and H. P. Yang, " $10 \mathrm{~Gb} / \mathrm{s}$ single-mode vertical-cavity surfaceemitting laser with large aperture and oxygen implantation," Semicond. Sci. Technol., vol. 19, pp. L86-L89, 2004.

[8] A. Furukawa, S. Sasaki, M. Hoshi, A. Matsuzono, K. Moritoh, and T. Baba, "High-power single-mode vertical-cavity surface-emitting lasers with triangular holey structure," Appl. Phys. Lett., vol. 85, no. 22, pp. 5161-5164, 2004.

[9] H. P. D. Yang, I-C. Hsu, F.-I. Lai, H. C. Kuo, and J. Y. Chi, "Highpower single-mode vertical-cavity surface-emitting lasers with multi-leaf holey structure," Jpn. J. Appl. Phys., vol. 45, no. 445, pp. L871-L873, 2006.

[10] D.-S. Song, S.-H. Kim, H.-G. Park, C.-K. Kim, and Y.-H. Lee, "Singlefundamental-mode photonic-crystal vertical-cavity surface-emitting lasers," Appl. Phys. Lett., vol. 80, no. 21, pp. 3901-3903, 2002.

[11] N. Yokouchi, A. J. Danner, and K. D. Choquette, "Etching depth dependence of the effective refractive index in two-dimensional photoniccrystal-patterned vertical-cavity surface-emitting laser structures," Appl. Phys. Lett., vol. 82, no. 9, pp. 1344-1346, 2003.

[12] H. P. D. Yang, F.-I. Lai, Y. H. Chang, H. C. Kuo, H. C. Yu, C. P. Sung, S. C. Wang, S. Y. Lin, and J. Y. Chi, "Single mode (SMSR > $40 \mathrm{~dB}$ ) protonimplanted photonic crystal vertical-cavity surface-emitting lasers," Inst. Electr. Eng. Electron. Lett., vol. 41, pp. 326-328, 2005.

[13] S. S. Mikhrin, A. E. Zhukov, A. R. Kovsh, N. A. Maleev, V. M. Ustinov, Yu. M. Shernyakov, I. P. Soshnikov, D. A. Livshits, I. S. Tarasov, D. A. Bedarev, B. V. Volovik, M. V. Maximov, A. F. Tsatsul'nikov, N. N. Ledentsov, P. S. Kop'ev, D. Bimberg, and Zh. I. Alferov, "0.94 $\mu$ m diode lasers based on Stranski-Krastanow and sub-monolayer quantum dots," Semicond. Sci. Technol., vol. 15, pp. 1061-1064, 2000.

[14] A. E. Zhukov, A. R. Kovsh, S. S. Mikhrin, N. A. Maleev, V. M. Ustinov, D. A. Livshits, I. S. Tarasov, D. A. Bedarev, M. V. Maximov, A. F. Tsatsul'nikov, I. P. Soshnikov, P. S. Kop'ev, Zh. I. Alferov, N. N. Ledentsov, and D. Bimberg, "3.9 W CW power from sub-monolayer quantum dot diode laser," Electron. Lett., vol. 35, no. 21, pp. 1845-1847, Oct. 1999.

[15] F. Hopfer, A. Mutig, M. Kuntz, G. Fiol, D. Bimberg, N. N. Ledentsov, V. A. Shchukin, S. S. Mikhrin, D. L. Livshits, I. L. Krestnikov, A. R. Kovsh, N. D. Zakharov, and P. Werner, "Single-mode submonolayer quantum-dot vertical-cavity surface-emitting lasers with high modulation bandwidth," Appl. Phys. Lett., vol. 89, pp. 141106-1-141106-3, 2006.

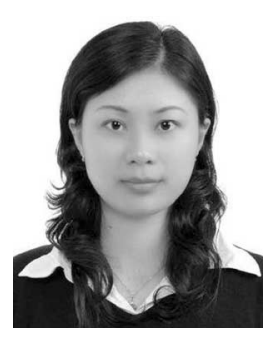

Fang-I. Lai received the B.S. degree in physics from Tung-Hai University,Taichung, Taiwan, R.O.C., in 1998, and the M.S. and Ph.D. degrees from the Institute of Electro-Optical Engineering, National Chiao-Tung University, Hsinchu, Taiwan, in 2001 and 2005, respectively.In 2007, she joined the Department of Electrical Engineering, Yuan Ze University, Taoyuan, Taiwan, as a Faculty Member. Her current research interests include semiconductor lasers, vertical-cavity surface-emitting lasers, blue and UV lasers, LED quantum-confined optoelectronic structures, optoelectronic materials, and semiconductor laser applications. 


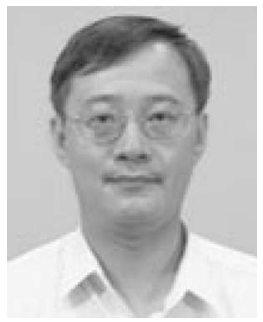

H. P. D. Yang received the B.S. degree in physics and the M.S. degree in electrical engineering from the National Tsing Hua University, Hsinchu,Taiwan, R.O.C., in 1985 and 1987, respectively, and the Ph.D. degree in electrical engineering from the University of Michigan, Ann Arbor, in 1993.

In 1994, he joined the Nanophotonic Centre, Industrial Technology Research Institute, Hsinchu, Taiwan. His current research interests include semiconductor lasers, vertical-cavity surface-emitting lasers (VCSELs), and photonic crystal VCSELs and LEDs. He is the author or coauthor of nearly 100 papers.

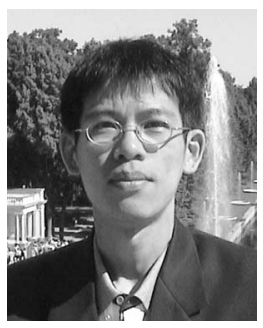

G. Lin received the B.S. degree in electronics engineering, the M.S. and Ph.D. degrees in electronics from the National Chiao-Tung University, Hsinchu, Taiwan, R.O.C., in 1994, 1995 and 2001, respectively.

He joined the Opto-Electronics and Systems Laboratories, Industrial Technology Research Institute, Hsinchu, for the substitute military service. His current research interests include molecularbeam epitaxy growth of GaAs-based compound semiconductor materials, design and fabrication of semiconductor lasers, as well as characterization and analysis of optoelectronic devices, research and development of GaAs-based long-wavelength semiconductor lasers, inclusive of InAs quantum dot lasers, quaternary GaInAsN and quinary GaInAsNSb quantum well lasers, both edge-emitting and surface-emitting.

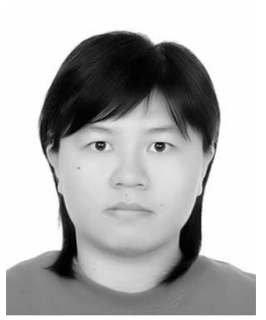

I.-Chen Hsu received the B.S. degree in physics from the National Tsing Hua University, Hsinchu, Taiwan, R.O.C., in 2004 and the M.S. degree in electro-optical engineering from the National Chiao Tung University, Hsinchu, in 2006.

Her current research interest include characterizing long-wavelength vertical-cavity surface-emitting lasers.

Jui-Nung Liu received the B.S. degree in materials science and engineering from the National Chiao Tung University, Hsinchu, Taiwan, R.O.C., in 2003, where he is currently working toward the M.S. degree at the Semiconductor Laser Technology Laboratory, Institute of Electro-Optical Engineering.

His current research interests include characterizing the high speed verticalcavity surface-emitting lasers.

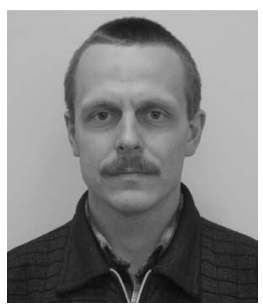

N. A. Maleev was born in Leningrad, Russia, in 1966. He graduated from the Leningrad Engineering Institute, Leningrad, Russia, in 1989, and received the Ph.D. degree in semiconductor physics from Saint Petersburg Electrotechnical University, St. Petersburg, in 1997.

In 1989, he joined Svetlana Co., St. Petersburg, where he was engaged on the microwave metelsemiconductor field effect transistor and psuedomorphic high electron mobility transistor. He joined A. F. Ioffe Physico-Technical Institute, Russian Academy of Sciences, St. Petersburg, in 1998, where he is currently a Senior Scientist. Since 2001, he has been a part-time Associated Professor with the Saint Petersburg Electrotechnical University. His current research interests include the development of novel III/V compound semiconductor device structures, including vertical-cavity surface emitters, InGaAs quantum dot and InGaAsN lasers, microwave transistors, and vertical-cavity surface-emitting lasers with quantum dot active region. He has coauthored more than 90 papers, and holds two patents.

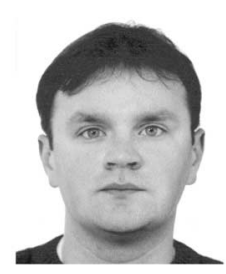

S. A. Blokhin was born in Chita, Russia, in 1979. He received the B.Sc. and M.Sc. degrees in physics and technology from St. Petersburg State Technical University, St. Petersburg, Russia, in 2000 and 2002, respectively, and the $\mathrm{Ph} . \mathrm{D}$. degree in semiconductor physics from A. F. Ioffe Physico-Technical Institute, Russian Academy of Science, St. Petersburg, in 2006.

Since 2002, he has been with A. F. Ioffe PhysicoTechnical Institute. He was with the Industrial Technology Research Institute, Hsinchu, Taiwan, R.O.C., in 2005, where he worked on processing and characterization of the oxide-confined quantum dot vertical-cavity surface-emitting lasers. His current research interests include development and optical studies of novel optoelectronic devices such as microdisk and vertical-cavity surfaceemitting lasers based on InGaAs quantum dots, photonic crystals, and singlephoton light sources. He has coauthored more than 25 papers.

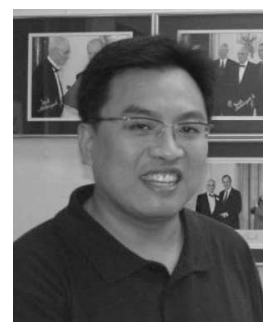

H. C. Kuo (M'99-SM'94) received the B.S. degree in physics from the National Taiwan University, Taipei, Taiwan, R.O.C., in 1990, the M.S. degree in electrical and computer engineering from Rutgers University, Newark, NJ, in 1995, and the Ph.D. degree in electrical and computer engineering from the University of Illinois, Urbana Champaign, in 1998

He has an extensive professional career both in research and industrial research institutions that includes Research Consultant in Lucent Technologies, Bell Laboratories, Murray Hill, NJ (1995-1997), R\&D Engineer in Fiber-Optics Division at Agilent Technologies, San Jose, CA (1999-2001), and R\&D Manager in LuxNet Corporation (2001-2002). He joined the Institute of Electro-Optical Engineering, National Chiao Tung University, Hsinchu, Taiwan, in 2002, as a Faculty Member. His current research interests include the epitaxy, design, fabrication, and measurement of high-speed InP-and GaAs-based vertical-cavity surface-emitting lasers, as well as $\mathrm{GaN}$ based-lighting emitting devices and nanostructures. He has authored or coauthored over 100 scientific papers and 100 international conference papers.

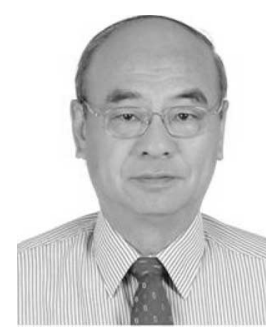

Jim Y. Chi (SM'97) received the B.S degree in physics from the National Tsinghua University, Hsinchu, Taiwan, R.O.C., in 1971 and the Sc.D. degree in material science from Massachusetts Institute of Technology, Cambridge, in 1979.

$\mathrm{He}$ is currently working with the Nanophotonic Centre, Industrial Technology Research Institute, Hsinchu, Taiwan, as the Director and a Projector Leader for advanced light sources for the next generation of optoelectronics systems, including the longwavelength vertical-cavity surface-emitting laser and laser diodes using InGaNAs materials, the GaN blue and green lasers, and the $980 \mathrm{~nm}$ high-power lasers for optical amplifier applications. He is also the Technical Leader of nanotechnology projects on quantum dots and photonics crystals. He has authored or coauthored more than 200 papers published in several journals and conference procedings. He has given many invited talks at conferences and institutions.

Dr. Chi received the 2004 Distinguished Engineers Award given by the Association of Chinese Engineers, Taiwan. 